\title{
Sapling and Handling of COVID-19 Patients in India
}

\author{
Nihar Gupta $^{1 *}$, Shweta Bahadure ${ }^{1}$, Obaid Noman ${ }^{1}$ and Arvind Bhake ${ }^{2}$ \\ ${ }^{1}$ Assistant Professor, Department of Pathology, Jawaharlal Nehru Medical College, India \\ ${ }^{2}$ Professor, Department of Pathology, Jawaharlal Nehru Medical College, India
}

*Corresponding author: Nihar Gupta, Assistant Professor, Department of Pathology, Jawaharlal Nehru Medical College, 17/221, Behind Geeta Lodge, Telephone Exchange Road, Bilaspur, Chhattisgarh, India, Tel: 7888044701

\begin{abstract}
This is a review article on how we can sample the COVID-19 patients, what samples can be obtained, what are the procedures to collect those samples and the precautions some laboratory personnel need to take while processing the samples. This review article mainly focuses on the various methods used in India while sampling COVID-19 patients. For now, RT-PCR is the most reliable test for COVID-19 detection. Few rapid test kits were introduced by ICMR, but now have been withdrawn as they were showing incorrect results at temperature more than 20 degree Celsius. WHO has labelled COVID-19 as a pandemic. No vaccine has been prepared, till date of writing this article which can be a cure. So for now, the only cure is prevention and protection. In India the front line tool for prevention is social distancing which has been achieved to an extent with lockdown throughout the country.
\end{abstract}

\section{Keywords}

COVID-19, Sampling of COVID-19 patients, Handling and transport of COVID-19 patients

\section{Background}

COVID-19 is an infectious disease caused by severe acute respiratory syndrome coronavirus 2 (SARSCoV-2). WHO has declared COVID-19 as pandemic and when I am writing this article more than 5 million patients have been reported worldwide, with India having around 52,000 cases. The first case of COVID-19 in India was reported on 30 Jan 2020, originating from China. As of 15 May 2020, the ministry of health and family welfare have confirmed a total of 81,970 cases, with 27,920 recoveries, and 2,649 deaths in India. December 2019 was the time when the outbreak of novel coronavirus associated respiratory disease came in front of the world, and later it was reported by WHO as COVID-19.
COVID-19 is a disease caused by a new strain of coronavirus, where 'CO' stands for corona, ' $\mathrm{VI}$ ' for virus and ' $D$ ' for disease, and ' 19 ' as it happened in 2019. Other names of COVID-19 are 2019-nCoV acute respiratory disease, Novel coronavirus [1-8].

On March 22 India observed a 14 hours' voluntary public curfew at the instance of the Prime Minister Narendra Modi ji. The Ministry of Electronics and Information Technology launched a smart phone application called Arogya Setu to help in "Contact tracing and containing the spread" of COVID-19 in India [1-8].

Despite the phylogenetic similarities among SARSCoV-2, severe acute respiratory syndrome coronavirus (SARS-CoV), and Middle East respiratory syndrome coronavirus(MERS-CoV), the difference in the clinical characteristics, such as greater transmissibility, relatively lower frequency of G.I symptoms, more patients with mild symptoms, and fewer fatalities, distinguish COVID-19. COVID-19 patients present in several ways: as asymptomatic infection, and as respiratory symptoms ranging from mild to severe pneumonia [1-8].

There are several coronaviruses which infects humans are: HCoV-229E, HCoV-NL63, HCoV-HKU1, and $\mathrm{HCOV}-\mathrm{OC} 43$; but these causes only mild respiratory disease. MERS-CoV and SARS-CoV have higher fatality rate. Now the present coronavirus was initially tentatively named 2019 novel coronavirus (2019-nCoV), and this virus is now named as SARS-CoV-2 by International Committee of Taxonomy of Viruses (ICTV) [9,10].

To reduce the risk during laboratory practice, it is important to use standard biological safety, regular training of staff, and use of standard training procedures.

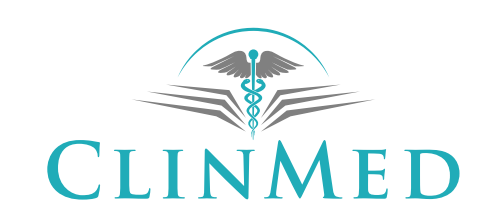

INTERNATIONAL LIBRARY

Citation: Gupta N, Bahadure S, Noman O, Bhake A (2020) Sapling and Handling of COVID-19 Patients in India. Int J Virol AIDS 7:066. doi.org/10.23937/2469-567X/1510066

Received: May 28, 2020: Accepted: June 25, 2020: Published: June 27, 2020

Copyright: (c) 2020 Gupta N, et al. This is an open-access article distributed under the terms of the Creative Commons Attribution License, which permits unrestricted use, distribution, and reproduction in any medium, provided the original author and source are credited. 


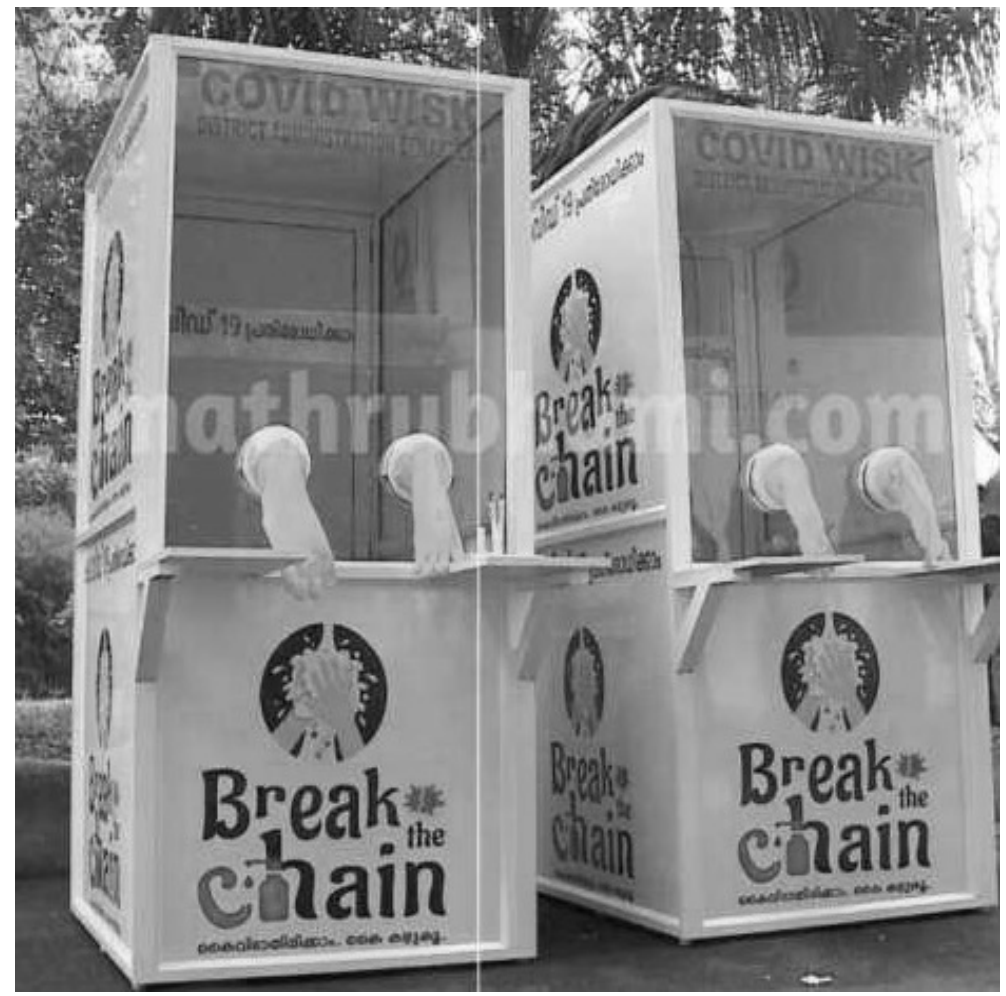

Figure 1: Special rooms designed for nasopharyngeal swab sampling which reduces the risk for health workers.

Risk assessment can be measured by: the procedure performed, identification of hazards involved in the process and procedure, how competent are the personnel to perform the procedures, the laboratory equipment and the resources available [11].

Nucleic acid detection is the gold standard for diagnosis of coronavirus disease 2019 , and the nasopharyngeal swab is the main method of sampling. This test shows $>94.7 \%$ sensitivity and $>98.6 \%$ specificity. As a result of psychological fear and due to irregular operation, the quality of nasopharyngeal swab collection is different and this leads to false negative and false positive, which affects the judgment of patient's condition. During the early days of COVID-19 infection, samples were collected in open environment. During sampling process, droplets and aerosol produced by sneezing, coughing, and talking of patients and close contact between health workers. Now special rooms have been designed for nasopharyngeal swab sampling which reduces the risk for health workers [12] (Figure 1).

\section{Specimen Types For COVID-19}

Diagnostic testing for COVIS-19, CDC recommends collecting and testing an upper respiratory specimen. The following samples are taken:

1. A nasopharyngeal specimen

2. An oropharyngeal specimen

3. A nasal mid-turbinate swab

4. An anterior nasal swab

5. Nasopharyngeal wash specimen
Swab should be placed quickly into sterile transport medium containing 2-3 $\mathrm{ml}$ of either viral transport medium, Amies transport medium, or sterile saline, unless the test is to be analysed directly [12].

Testing the lower respiratory tract can also be done. Patients who produce productive cough, sputum can be collected and tested for COVID-19. So the samples for lower respiratory tract are [12]:

1. Sputum

2. Aspirate

3. Lavage

\section{Methods of Specimen Collection}

Patients needs to be informed about the process of sampling and what can be the possible adverse events that can take place possibly and a written informed consent needs to be taken. Patients should be asked to avoid eating and drinking anything before sampling [12] (Figure 2 and Figure 3).

For children who may not cooperate well, the specimen collection is performed with children in supine position, a family member helps to support the chin and collector collects the specimen while pressing the child's forehead [12].

\section{Nucleic acid amplification tests (NAAT) for COVID-19 virus}

Routine confirmation of cases of COVI-19 is by detection of unique sequences of virus RNA by NAAT such as real-time reverse transcription polymerase chain re- 


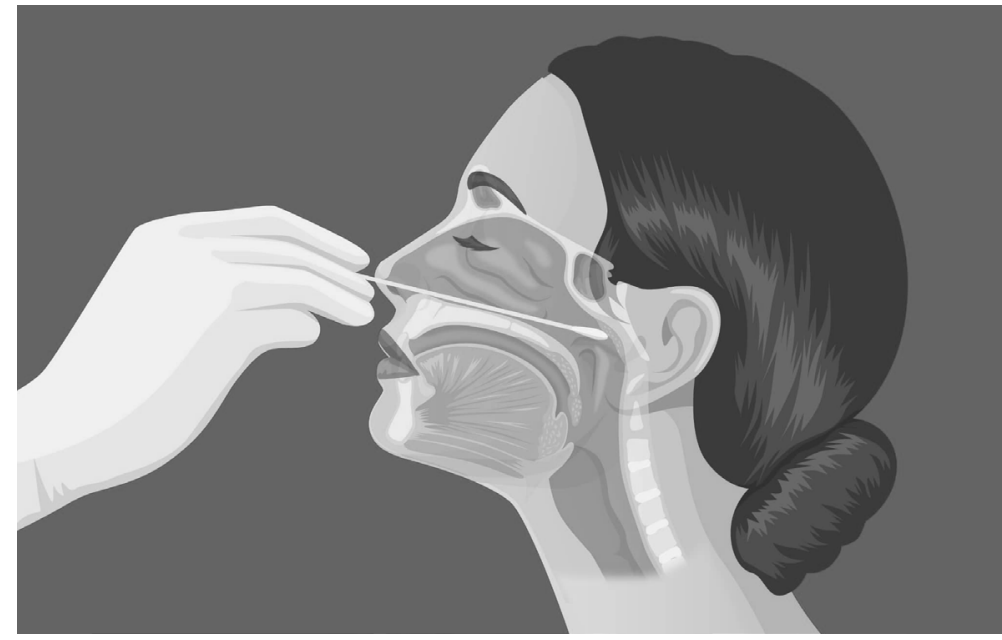

Figure 2: For collecting a nasal swab, a polyester swab with a shaft should be inserted carefully into the nose until reaching the nasal palate and stay there for 15-30 seconds, twist 3-4 times and carefully remove it. The same procedure should be repeated from another naris with another swab. These two swabs should be placed in a sterile tube containing $3 \mathrm{ml}$ viral transport medium or sterile saline.

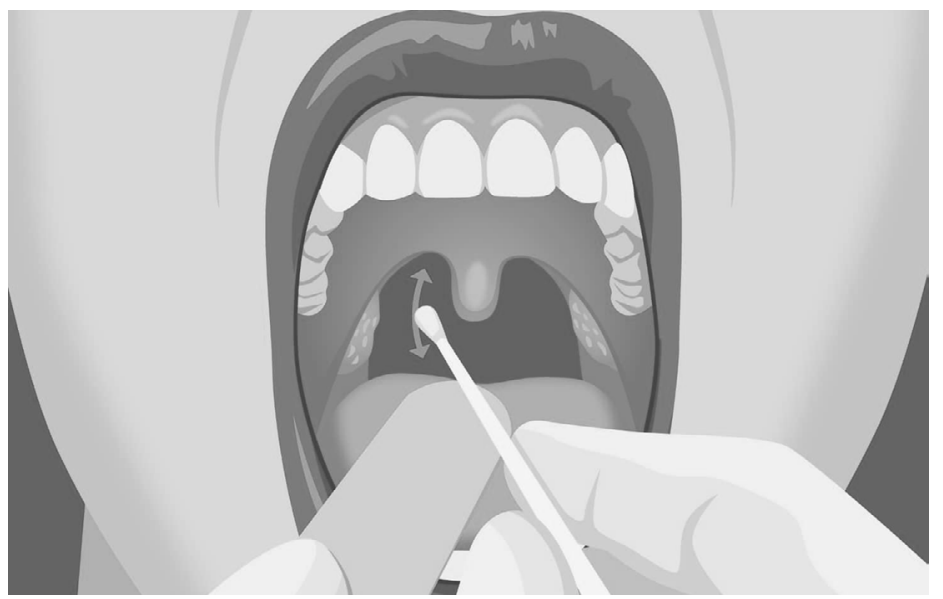

Figure 3: When collecting an oropharyngeal swab, the practitioner holds the swab in his/her right hand, then swabs the bilateral tonsils and the posterior pharyngeal wall. While removing the mucosa of tongue and oral mucosa should not be touched. The swab should be placed in a sterile tube containing $3 \mathrm{ml}$ viral transport medium or sterile saline.

action (rRT-PCR) with confirmation by nucleic acid sequencing when required. The viral genes targeted so far are N, E, S and RdRP genes. Heat treatment of samples should not be done prior to RNA extraction [13-15].

\section{Laboratory confirmation of cases by NAAT in areas with no known COVID-19 virus circulation}

To consider a case as laboratory-confirmed by NAAT in an area with no COVID-19 virus circulation, one of the following conditions need to be met [13-15]:

- A positive NAAT result for at least two different targets on the COVID-19 virus genome, of which at least one target is preferably specific for COVID-19 virus using a validated assay; $O R$

- One positive NAAT result for the presence of beta coronavirus, and COVID-19 virus further identified by sequencing partial or whole genome of the virus as long as the sequence target is larger or different from the amplicon probed in the NAAT assay used.

\section{Laboratory confirmed cases by NAAT in areas with an established COVID-19 virus circulation}

In areas where COVID-19 virus is widely spread a simpler algorithm can be adopted where screening by rRT-PCR is considered sufficient and no further testing is required.

One or more negative results do not rule out the possibility of COVID-19 virus infection. A number of factors could lead to a negative result in an infected individual, including:

- Poor quality of the specimen, containing little patient material (as a control, consider determining whether there is adequate human DNA in the sample by including a human target in the PCR testing)

- The specimen was collected late or very early in the infection

- The specimen was not handled and shipped appropriately 
- Technical reasons inherent in the test, e.g. virus mutation or PCR inhibition.

If a negative result is obtained from a patient with a high index of suspicion for COVID-19 virus infection, particularly when only upper respiratory tract specimens were collected, additional specimens, including from the lower respiratory tract if possible, should be collected and tested [13-15].

\section{Rapid test kit}

Initially, the rapid test kits were introduced by ICMR, which were supposed to be used as screening test for COVID-19 patients. Guidelines on Rapid test kits-

- Can be done on blood, serum or plasma samples.

- Test results are available within 30 mins.

- Test comes positive after 7-10 days of infection.

- Positive test result indicates exposure to SARS-CoV-2.

- Negative test doesn't rule out COVID-19 infection.

Later, it was claimed that the rapid test kits were not showing correct results and the ICMR authorities said it could be possible because the kits have not been standardised. These kits had the approval of the US Food and Drug Administration, but it was learnt that these kits show error results when kept above 20 degree Celsius [13-15]

\section{Viral sequencing}

In addition to provide confirmation of the presence of COVID-19 virus, regular sequencing of a percentage of specimens from clinical cases is useful in monitoring viral genome mutations that equally affects the performance of medical treatment and diagnostic tests [1315].

\section{Viral culture}

Viral isolation is not recommended as a routine diagnostic procedure. Culture-based systems for virus isolation have been the "gold standard" in clinical virology for decades and have served the laboratory well when there was little else available for the diagnosis of viral illnesses. However, there are many legitimate reasons why the use and relative importance of virus culture are declining with the continued development of rapid and accurate molecular tests [13-16].

In general, the isolation of viruses in culture is slow, time-consuming, and labor-intensive and lacks the sensitivity needed to have an appreciable impact on clinical decision making. Many clinically relevant viruses are simply difficult to grow or cannot be grown at all in cultured cells, while other viruses require specialized culture systems that are either not available or too complicated for routine use in diagnostic laboratories. Traditional tube cultures, although viewed as being comprehensive in growing a wide range of viruses and capable of detecting unsuspected new viruses or more common viruses in new places, fail to isolate viruses in many instances and can take days to weeks to provide a final result. While centrifugation-assisted cultures using individual, mixed, or genetically engineered cell lines are designed to be faster and more user-friendly than tube cultures, they are not always as sensitive and are normally limited by the quality and availability of reagents and the number and types of cell lines that can be used to grow a variety of different viruses. Normally, only viruses that are being sought after or for which the cell lines are designed can be identified and only one or a few viruses can be detected at a time.

Still viral culture still remains a major tool for detection of COVID-19 but are not being used as a routine test.

\section{Serological testing}

Serological surveys can aid investigation of an ongoing COVID-19 outbreak and retrospective assessment of the extent of an outbreak. In cases where NAAT assays are negative and there is strong epidemiological link to COVID-19 infection, paired serum samples could support diagnosis once validated serology tests are available. We can store serum samples for this purposes.

Paired samples are necessary for confirmation with initial sample collected in the first week of illness and the second ideally collected 2-4 weeks later (optimal timing for convalescent sample needs to be established). Baseline serum taken as early as possible within incubation period of contact [13-15].

\section{Packaging and transport}

Specimens should reach the laboratory as soon as possible. At 2-8 degree Celsius the specimen should be stored and transported. Specimen may be frozen at -20 to -70 degree and can be shipped on dry ice if there is further delay.

Packaging and transport of contagious samples, may be performed at containment level 2 if the specimens are already contained within a sealed and decontaminated primary container. Cultured samples for research or calibration must be transported in accordance with category a transportation regulation [17] (Table 1).

\section{Costing of test}

As per the guidelines of Supreme Court of India, the maximum cost of test sample cannot exceed Rs 4,500 (60 Dollars) in private laboratories. This includes Rs 1,500 (20 Dollars) as screening test for suspected cases, and an additional Rs 3,000 (40 Dollars) for confirmation of test. In government setup these tests are being done free of cost. The reason for involving private laboratories in India was the increasing load of tests. Still India could only achieve $1,00,000$ test per day which is quite less than the targeted numbers. The data of number of 
Table 1: Table shows various types of specimens taken for COVID-19 patients and their appropriate temperature for transport and storage.

\begin{tabular}{|l|l|l|l|}
\hline Specimen Type & Collection Material & Transport to Laboratory & Storage Until Testing \\
\hline $\begin{array}{l}\text { Nasopharyngeal and } \\
\text { Oropharyngeal Swabs }\end{array}$ & $\begin{array}{l}\text { Dacron or polyester flocked } \\
\text { swabs }\end{array}$ & $2-8{ }^{\circ} \mathrm{C}$ & $<5 \mathrm{~d}, 2-8^{\circ} \mathrm{C}$ \\
$>5 \mathrm{~d},-70^{\circ} \mathrm{C}(\mathrm{dry}$ ice)
\end{tabular}

Table 2: Table shows Form used in laboratories to be submitted with sample.

\section{Submitter Information}

Name of submitting hospital, laboratory or other facility

Physician

Address

Phone number

Case definition

O Suspected

O Probable case

First Name

Patients ID number

Address

Patients Information

Specimen Information

Type of Specimen

O Nasopharyngeal and oropharyngeal swab

O Bronchoalveolar lavage

O Endotracheal aspirate

O Nasopharyngeal aspirate

O Nasal wash

O Sputum

O Sputum

O Serum

O lung tissue

O Whole blood

O Stool

Please tick the box if sample taken is postmortem

Has patient has history of travelling to affected area

Clinical details

Has the patient had contact with confirmed cases?

\begin{tabular}{|l|l|}
\hline O Yes & O Country: \\
O No & O Return date: \\
\hline O Yes & O Unknown \\
O No & O Other exposure \\
\hline
\end{tabular}

Additional information 
test done in private and government sectors separately is not available.

\section{Laboratory test request form for COVID-19}

For submitting a sample to laboratory for COVID-19 following form need to be filled and submitted along with sample. The form contains columns for basic information of patient so that patient can be contacted and traced for follow-up (Table 2).

\section{References}

1. Gorbalenya A, Baker S, Baric R, de Groot R, Drosten C, et al. (2020) Coronaviridae study group of the international committee on taxonomy of viruses. The species severe acute respiratory syndrome-related coronavirus: Classifying 2019-nCoV and naming it SARS-CoV-2. Nature Microbiology 2020: 3-4.

2. World Health Organization (2019) Coronavirus disease (COVID-2019) situation reports.

3. Kinross P, Suetens C, Dias JG, Alexakis L, Wijermans A, et al. (2020) Rapidly increasing cumulative incidence of coronavirus disease (COVID-19) in the European Union/European economic area and the United Kingdom, 1 January to 15 March 2020. Euro surveill 25: 2000285.

4. Wu Z, McGoogan JM (2020) Characteristics of and important lessons from the coronavirus disease 2019 (COVID-19) outbreak in China: summary of a report of 72314 cases from the Chinese center for disease control and prevention. JAMA 323: 1239-1242.

5. Guan WJ, Ni ZY, Hu Y, Liang WH, Ou CQ, et al. (2020) Clinical characteristics of coronavirus disease 2019 in China. N Engl J Med 382: 1708-1720.

6. Wang Y, Wang Y, Chen Y, Qin Q (2020) Unique epidemiological and clinical features of the emerging 2019 novel coronavirus pneumonia (COVID-19) implicate special control measures. J Med Virol 92: 568-576.
7. Rothe C, Schunk M, Sothmann P, Bretzel G, Froeschl G, et al. (2020) Transmission of 2019-nCoV infection from an asymptomatic contact in Germany. N Engl J Med 382: 970-971.

8. Huang C, Wang Y, Li X, Ren L, Zhao J, et al. (2020) Clinical features of patients infected with 2019 novel coronavirus in Wuhan, China. Lancet 395: 497-506.

9. Zhu N, Zhang D, Wang W, Li X, Yang B, et al. (2020) A novel coronavirus from patients with pneumonia in China, 2019. N Engl J Med 382: 727-733.

10. Gorbalenya AE, Baker SC, Baric R, Groot RJ, Drosten C, et al. (2020) Severe acute respiratory syndrome-related coronavirus: The species and its viruses-a statement of the Coronavirus Study Group. bioRxiv.

11. Habib ZH, Alam AN (2020) Biosafety measures in the laboratory during handling of specimens of suspected COVID-19 patients. Bangladesh Journal of Infectious Diseases 24: S66-S68.

12. Qian Y, Zeng T, Wang H, Xu M, Chen J, et al. (2020) Safety management of nasopharyngeal specimen collection from suspected cases of coronavirus disease 2019. International Journal of Nursing Sciences 7: 153-156.

13. Meyer B, Drosten C, Müller MA (2014) Serological assays for emerging coronaviruses: Challenges and pitfalls. Virus Res 194: 175-183.

14. World Health Organization (2020) Laboratory testing for coronavirus disease 2019 (COVID-19) in suspected human cases: Interim guidance.

15. Xiao SY, Wu Y, Liu H (2020) Evolving status of the 2019 novel coronavirus infection: Proposal of conventional serologic assays for disease diagnosis and infection monitoring. J Medical Virol 92: 464-467.

16. Leland DS, Ginocchio CC (2007) Role of cell culture for virus detection in the age of technology. Clinical Microbiol Rev 20: 49-78.

17. World Health Organization (2020) Laboratory biosafety guidance related to the novel coronavirus (2019-nCoV). Interim guidance. 